\title{
Postoperative Analgesia Following Caesarean Section: Intravenous Patient Controlled Analgesia versus Conventional Continuous Infusion
}

\author{
Samina Ismail, Gauhar Afshan, Abdul Monem, Aliya Ahmed \\ Department of Anaesthesia, The Aga Khan University, Karachi, Pakistan. \\ Email: samina.ismail@aku.edu
}

Received July $2^{\text {nd }}$, 2012; revised August $3^{\text {rd }}, 2012$; accepted August 23 ${ }^{\text {rd }}, 2012$

\begin{abstract}
Background: Management of postoperative pain after caesarean section (CS) requires a balance between pain relief and undesirable side effects of drugs and technique. In order to improve postoperative pain management after caesarean section, we compared intravenous patient controlled analgesia (IV-PCA) with our current hospital practice, which is continuous opioid infusion. Method: We enrolled one hundred and twenty patients in our prospective randomized trial after an uneventful elective caesarean section under spinal anaesthesia. All patients received $0.5 \mathrm{mg} / \mathrm{kg}$ bolus of pethidine on first complaint of pain or at 120 minutes after institution of spinal anaesthesia. Depending upon the randomization, Group P received IV-PCA with $0.15 \mathrm{mg} / \mathrm{kg}$ bolus pethidine with 10-minute lockout and Group C received continuous pethidine infusion at a rate of $0.15 \mathrm{mg} / \mathrm{kg} / \mathrm{hr}$. Statistical Analysis: For qualitative variables means and standard deviations were computed and analyzed by T-test, Mann Whitney U test and repeated measures ANOVA. Frequency and percentages were computed for qualitative data and analyzed by Chi-Square and Fischer exact test. A p-value of less than 0.05 was treated as significant. Results: The numeric rating score for pain, need for rescue analgesia and incidence of nausea and vomiting was significantly lower (p-value $<0.001$ ) in IV-PCA group as compared to continuous infusion group at 6,12 and 24 hours postoperatively, 98\% of the patients were satisfied with pain management in Group P as compared to 70\% ( $<0.001)$ in Group C. Conclusion: Our results showed improved pain control, less need for rescue analgesia for breakthrough pain, lower incidence of nausea and vomiting and greater patient satisfaction with IV-PCA. In the absence of preservative free narcotics for intrathecal use, postoperative pain management can be significantly improved by using IV-PCA instead of continuous opioid infusion in patients undergoing caesarean section.
\end{abstract}

Keywords: Postoperative Pain; IV-PCA; Continuous Opioid Infusion; Opioids; Caesarean Section

\section{Introduction}

Caesarean section (CS) commonly induces moderate to severe pain for about 48 hours. It requires optimal perioperative pain management; which not only allows the mother to ambulate early but also plays a key role in the postoperative rehabilitation after CS [1].

Intravenous patient controlled analgesia (IV-PCA) is now widely used in clinical practice, and is among the most recommended techniques for the control of moderate to severe postoperative pain [2]. The devices used for IV-PCA are expensive and material costs per patients are usually higher compared with conventional analgesia [3]. Due to cost constraints, there is limited availability of IV-PCA devices in our hospital; therefore we have been using continuous opioid infusion as the alternate cost effective method for most of our CS patients.
Several studies have compared the analgesic effects of different opioids using IV-PCA [4] and use of pethidine in patient controlled epidural analgesia (PCEA) versus IV-PCA [5]. However in spite of extensive literature search; we did not find any study which has compared continuous infusion and IV-PCA with pethidine in post caesarean patients.

In our hospital setting there is a general perception among surgeons that adequate pain relief is achieved via continuous infusion. Our study question was to observe if our current hospital practice of pain management, which is continuous opioid infusion is providing adequate pain relief or needs to be replaced by some advanced modality like IV-PCA in patients undergoing elective caesarean section. Therefore the primary objective of this prospective randomized study was to assess if there are any clinical benefits of using IV-PCA compared to continu- 
ous opioid infusion using pethidine in terms of pain score. Our secondary objectives were to compare opioid consumption, need for rescue analgesia, patient satisfaction and side effects between the two groups.

\section{Materials and Methods}

In this randomized controlled prospective study, we obtained approval from the hospital ethics committee, to study 120 American Society of Anesthesiologists (ASA) physical status I \& II parturient at term having healthy fetuses, undergoing elective caesarean section under spinal anaesthesia. Exclusion criteria were: patients allergic to study drugs, surgery exceeding more than 90 minutes, women having additional procedures like tubal ligation, women having chronic pain syndromes, on long term analgesic medications and patients refusing to participate in the study. All patients fulfilling the inclusion criteria and scheduled for elective CS under spinal anaesthesia were asked for written, informed consent before surgery. Once they consented to participate in the study and signed the written informed consent, they were instructed on numeric rating scale (NRS) for pain assessment with zero being no pain and 10 representing worst pain imaginable. As blinding was not possible in this study, we made sure that all assessment, data collection and documentation in the form was done by the acute pain management service (AMPS) nurse who was otherwise not a part of the study project. Primary outcome of our study was pain control assessed by numeric rating scale and secondary outcomes were opioid consumption, need for rescue analgesia and incidence of side effects. Assessments for pain (rest and movement) and of side effects were done according to the set APMS protocol of our hospital setting.

All patients received standard spinal anaesthesia in the sitting position with a 25 gauge pencil point spinal needle with introducer, after local infiltration of skin with 2 - 3 $\mathrm{ml}$ of $2 \%$ lignocaine. We used $10 \mathrm{mg}$ of $0.5 \%$ isobaric bupivacaine with $25 \mathrm{mcg}$ of fentanyl for intrathecal injection. Surgery was allowed to start after establishment of block up to T4 to T5 level. After successful completion of surgery, patients were shifted to recovery room (RR) and were monitored by a RR nurse. All patients who had uneventful surgery which finished within 90 minutes were enrolled in the study. All the enrolled patients had already given written informed consent before surgery. Patients were randomized by drawing shuffled coded envelopes to receive one of the two analgesic regimens. There were 120 coded sealed opaque envelopes which were prepared by the primary investigator. 60 envelops contained the code "IV-PCA" and 60 contained the code "Continuous Infusion". The envelopes were shuffled every time before drawing the envelope for each patient.

On patient's first complaint of pain, or 120 minutes after the institution of spinal anaesthesia, (whichever came first) a loading dose of $0.5 \mathrm{mg} / \mathrm{kg}$ of pethidine was given over 2 minutes. Depending upon the randomization, patients were either attached to IV-PCA pumps (Graseby 3300 PCA pumps manufactured by SIMS Graseby limited, Watford Herts UK) or continuous infusion by volumetric pumps (Volumed $\mu$ VP 5005 acromed Switzerland) after 15 minutes of the loading dose.

In continuous infusion group, pethidine infusion was started at a rate of $0.15 \mathrm{mg} / \mathrm{kg} / \mathrm{hour}$ and was continued for 24 hrs. In IV-PCA group, an IV-PCA pump was attached to the patient with no background infusion and bolus of $0.15 \mathrm{mg} / \mathrm{kg}$ body weight with lockout interval of 10 minutes.

NRS for pain was assessed by the nurse from the acute pain management service (APMS), who was otherwise not a part of the study project. NRS was assessed in two different ways; one at rest and the other at movement where patient was asked to bend her knees. Patient's pain was assessed on arrival in the RR, at 120 minutes of spinal anaesthesia and 5, 15 and 30 minutes after giving the bolus and attaching one of the two pain regimes by one of the investigator. When a patient fulfilled all discharge criteria, she was shifted to the ward where she was assessed for pain at 6, 12 and 24 hours by the acute pain management service (APMS). Besides the APMS, ward nurses checked and recorded the NRS every 4 hours as per hospital protocol. APMS and ward nurses were informed about the study protocol, data collection and documentation in the study form. The data collected was reviewed separately by two investigators on the first postoperative day for any missing entries and was submitted to the statistician for data entry.

Patients in both groups were instructed to call the nurse for rescue analgesia if pain relief was not adequate (NRS > 3). Rescue analgesia was provided according to the written orders for postoperative pain management. First line of rescue analgesia was pethidine $10 \mathrm{mg}$ IV stat (total $30 \mathrm{mg}$ ). If a patient still complained of pain after 30 minutes of three doses of $10 \mathrm{mg}$ of pethidine, second line of rescue analgesia was provided with $2 \mathrm{mg}$ of IV morphine. Rescue analgesia was repeated every 20 minutes, till the patient was completely pain free. All patients, irrespective of the group were assessed for the need of rescue analgesia and the drug, dose and timings were noted in the study form by the nurse from APMS.

Patients were assessed for side effects both in the RR and the ward. Postoperative nausea and vomiting (PONV) was measured with the help of scoring system used by the set APMS protocol, where 0 was no PONV, 1 was mild nausea on inquiry, 2 was nausea without inquiry, 3 
was vomiting and 4 was severe repeated vomiting. Sedation score ranged from 1 to 5 , where 1 was alert and 5 was denoted to patient in deep sleep. Pruritus, drop in oxygen saturation and respiratory rate less than 10 were other complications noted. These were recorded by the ward nurses, who were informed about the study protocol and its documentation. All adverse effects were to be managed according to hospital protocol and included in our results.

All patients were prescribed injection metoclopramide $10 \mathrm{mg} \mathrm{I} / \mathrm{V}$ stat 8 hourly and tablet paracetamol 1 gram three times a day and diclofenac suppository $100 \mathrm{mg}$ twice a day.

APMS team on the first postoperative day interviewed the patient for their satisfaction with postoperative pain management therapy and whether they would like to have the same pain management therapy for the future surgeries.

\section{Results}

We studied 120 patients, 60 in each group. The groups matched for age, weight, height, BMI and previous caesarean sections (Table 1). All patients received the same dose of intraoperative anaesthesia and analgesia (Bupivacaine $10 \mathrm{mg}$ and fentanyl $25 \mathrm{mcg}$ ) and the duration of all surgeries was less than 90 minutes. The surgical technique was same and uterus was exteriorized for stitching in all cases.

The NRS for pain at rest and movement in the RR was one or less for both groups and no significant differences were observed within groups or between groups at different times. The NRS at rest and movement for continuous infusion group at 6,12 and 24 hours postoperatively was significantly higher compared to IV-PCA group ( $<$ 0.001) (Figures 1 and 2). Number of patients in IV-PCA group and continuous infusion group having different severity of pain score measured at 6,12 and 24 hours in the postoperative period at rest and movement is shown in Tables 2 and $\mathbf{3}$. There was a gradual increase in NRS from 6 to 12 hours and then a gradual decline from 12 to 24 hours. None of the patients in either group had NRS of more than 7.

None of the patients in either group had drop in oxygen saturation or respiratory rate less than 10 . There was no difference in the incidence of vomiting; however incidence of nausea (score $1 \& 2$ ) was significantly higher between 6 and 12 hours in continuous infusion group compared to IV-PCA group $(\mathrm{p}<0.001)$.

In the IV-PCA group, nine (14.8\%) patients required rescue analgesia as compared to $42(70 \%)$ in continuous infusion group which was significantly higher as compared to IV-PCA group $(\mathrm{p}<0.001)$. The need for rescue analgesia was mostly between 6 - 12 hours postopera- tively. None of the patients in either group required morphine as pain was relieved with three doses or less of $10 \mathrm{mg}$ pethidine, every time rescue analgesia was required. A total of 13 patients required rescue analgesia more than once during the study period (2 patients from IV-PCA group and eleven patients were from continuous infusion group). In IV-PCA group, the mean of demand made on PCA was 36.2 (SD 11.91) and mean of successful demands were 31.52 (SD 10.89).

There was no significant difference between the total amounts of pethidine consumed over 24 hours between the two groups (360 mg in IV-PCA group versus $363 \mathrm{mg}$ in continuous group). Ninety eight percent of the patients were satisfied with pain management and wanted the same form of analgesia for future surgeries in the IVPCA group as compared to $70 \%$ in continuous infusion group $(\mathrm{p}<0.001)$.

Table 1. Characteristics of patients receiving pethidine via continuous infusion (Group C) or patient controlled intravenous technique (Group P). Values refer to mean (SD).

\begin{tabular}{cccc}
\hline Variables & Group C & Group P & p value \\
\hline Age (yrs) & $27(3.58)$ & $27(3.95)$ & 0.72 \\
Weight $(\mathrm{kg})$ & $71(6.79)$ & $72(6.32)$ & 0.43 \\
Height $(\mathrm{cm})$ & $159(5.26)$ & $158(5.38)$ & 0.25 \\
BMI $\left(\mathrm{kg} / \mathrm{m}^{2}\right)$ & $28(3.18)$ & $29(3.30)$ & 0.17 \\
Previous CS & $63 \%$ & $62 \%$ & 0.50 \\
\hline
\end{tabular}

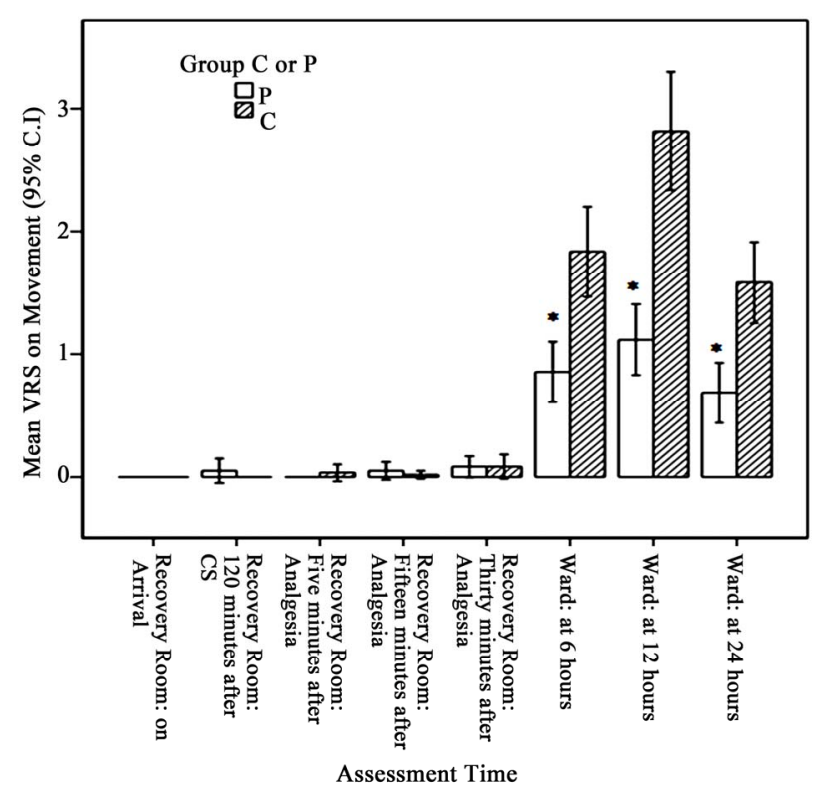

Figure 1. Verbal rating score (VRS) for pain on movement for Group P \& Group C for different times in the postoperative period ${ }^{*} \mathrm{p}<0.001$. 


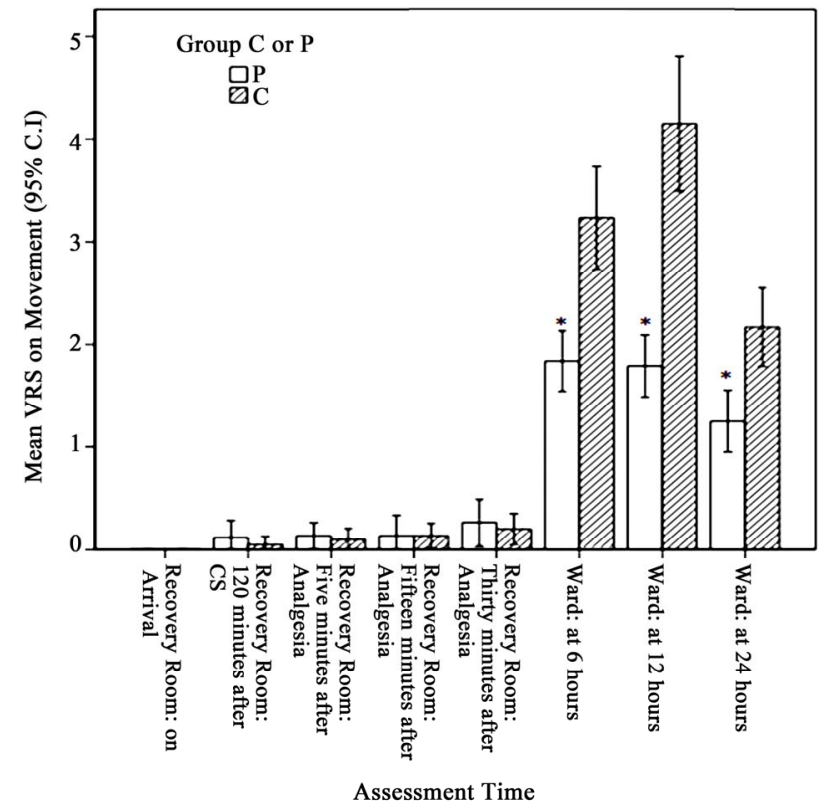

Figure 2. Verbal rating score (VRS) for pain on movement for Group $P$ \& Group $C$ for different times in the postoperative period ${ }^{*} \mathrm{p}<\mathbf{0 . 0 0 1}$.

Table 2. Number of patients (\%) having different pain scores on rest at 6,12 and 24 hours post operatively receiving pethidine via patient control intravenous (Group $P$ ) or continuous infusion (Group C) techniques.

\begin{tabular}{ccccc}
\hline $\begin{array}{c}\text { Post-Operative } \\
\text { Time (hrs) }\end{array}$ & $\begin{array}{c}\text { Pain Score } \\
(\text { NRS })^{\epsilon}\end{array}$ & $\begin{array}{c}\text { Group P } \\
(\mathrm{n}=60)\end{array}$ & $\begin{array}{c}\text { Group C } \\
(\mathrm{n}=60)\end{array}$ & p value \\
\hline \multirow{2}{*}{6 hrs } & NRS 1-3 & $60(100 \%)$ & $55(95 \%)$ & \\
& NRS 4-6 & $0(0 \%)$ & $5(5 \%)$ & 0.57 \\
& NRS 7 & $0(0 \%)$ & $0(0 \%)$ & \\
& NRS 1-3 & $60(100 \%)$ & $33(55.7 \%)$ & \\
12 hrs & NRS 4-6 & $0(0 \%)$ & $26(42.6 \%)$ & $<0.01^{*}$ \\
& NRS 7 $7^{\dagger}$ & $0(0 \%)$ & $1(1.6 \%)$ & \\
& NRS 1-3 & $60(100 \%)$ & $57(95 \%)$ & \\
24 hrs & NRS 4-6 & $0(0 \%)$ & $3(5 \%)$ & 0.244 \\
& NRS 7 & $0(0 \%)$ & $0(0 \%)$ & \\
\hline
\end{tabular}

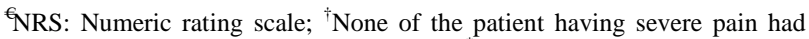
numeric rating score (NRS) of more than $7 ;{ }^{*} \mathrm{p}<0.05$.

\section{Discussion}

In this study we demonstrated that using pethidine by IVPCA gave better pain scores at 6,12 and 24 hours postoperatively compared to continuous intravenous pethidine infusion after elective caesarean section with an acceptable side effect profile. Although the pain scores were better and the need for rescue analgesia was less in
Table 3. Number of patients (\%) having different pain scores on movement at 6, 12 and 24 hours post operatively receiving pethidine via patient control intravenous (Group P) or continuous infusion (Group C) techniques.

\begin{tabular}{|c|c|c|c|c|}
\hline $\begin{array}{l}\text { Post-Operative } \\
\text { Time (hrs) }\end{array}$ & $\begin{array}{l}\text { Pain Score } \\
\text { (NRS) }^{\epsilon}\end{array}$ & $\begin{array}{l}\text { Group P } \\
(\mathrm{n}=60)\end{array}$ & $\begin{array}{l}\text { Group C } \\
(\mathrm{n}=60)\end{array}$ & $\mathrm{p}$ value \\
\hline \multirow{3}{*}{$6 \mathrm{hrs}$} & NRS 1-3 & 58 (96.7\%) & 32 (53.3\%) & \multirow{3}{*}{$\mathrm{p}<0.01^{*}$} \\
\hline & NRS 4-6 & 2 (3.3\%) & 27 (45\%) & \\
\hline & NRS $7^{\dagger}$ & $0(0 \%)$ & $1(1.7 \%)$ & \\
\hline \multirow{3}{*}{$12 \mathrm{hrs}$} & NRS 1-3 & 58 (96.7\%) & 29 (48.3\%) & \multirow{3}{*}{$\mathrm{p}<0.01^{*}$} \\
\hline & NRS 4-6 & 2 (3.3\%) & 25 (41.7\%) & \\
\hline & NRS $7^{\dagger}$ & $0(0 \%)$ & $6(10 \%)$ & \\
\hline \multirow{3}{*}{$24 \mathrm{hrs}$} & NRS 1-3 & 58 (96.7\%) & 55 (91.7\%) & \multirow{3}{*}{0.219} \\
\hline & NRS 4-6 & 2 (3.3\%) & 5 (8.3\%) & \\
\hline & NRS $7^{\dagger}$ & $0(0 \%)$ & $0(0 \%)$ & \\
\hline
\end{tabular}

${ }^{\epsilon}$ NRS: Numeric rating scale; ${ }^{\dagger}$ None of the patient having severe pain had numeric rating score (NRS) of more than $7 ;^{*} \mathrm{p}<0.05$.

IV-PCA group, the amount of pethidine consumed was similar in the two groups.

PCA works on the principle of individual variability of "what you need is what you get" (WYNIWYG) [6]. Since the total amount of pethidine consumed over 24 hours was the same in both groups, one explanation for improved pain control with PCA could be the timing of the doses. Patients' own control over their pain management may have allowed them to obtain an opioid bolus at the initial feeling of pain, without having to wait for the nurses and doctors to provide analgesia, resulting in overall improved analgesia. The optimal bolus dose in PCA is controversial. Owen et al. [7] studied the effect of different doses of morphine $(0.5,1 \mathrm{and} 2 \mathrm{mg}$ ) and found that $1 \mathrm{mg}(0.01-0.02 \mathrm{mg} / \mathrm{kg})$ was the optimal bolus PCA dose. Therefore we chose $0.15 \mathrm{mg} / \mathrm{kg}$ (average $10-15 \mathrm{mg}$ ) bolus dose of pethidine, considering an analgesic potency one tenth that of morphine.

Various studies [8-10] using continuous opioid infusion (either as a fixed dose or dose based on weight) have failed to identify an ideal dose that would provide adequate analgesia during rest and activity without supplemental bolus doses and prevent side effects [11]. We used a fixed dose of pethidine according to the weight of patient $(0.5 \mathrm{mg} / \mathrm{kg}$ bolus followed by $0.15 \mathrm{mg} / \mathrm{kg}$ continuous infusion per hour), as this is the routine practice and kept the option of rescue analgesia for patients having inadequate pain relief.

We found a statistically significant difference between the need for rescue analgesia between the two groups. The need for rescue analgesia was mostly between 6 - 12 
hours postoperatively. The NRS at rest and movement showed a rising trend and was found to be highest at 12 hours and then a declining trend was observed. Somewhat similar observations were made in the study of $\mathrm{Al}$ hashemi et al. [12] where they found a peak of morphine requirement between 4 - 8 hours. The gradual increase in NRS score and rescue analgesia during this time could be explained by the recession of spinal anaesthesia and patients' mobilization. This was the time when 8 patients complained of severe pain, all from continuous infusion group but none of the patients had NRS of more than seven. In all patients NRS came to 1 - 3 after 10 - 15 minutes of giving rescue analgesia and none of the patients required morphine which was our second line of rescue analgesia. However 13 patients did require rescue analgesia more than twice during the study period. We kept morphine as our second line of analgesic, largely because we needed a stronger analgesic for patients in whom pain would not be relieved by pethidine. As in our country morphine is only available in limited amount we could not use it as our primary analgesic agent.

Even with the increased use of rescue analgesia, the amount of pethidine consumed during the study period remained the same in the two groups. This is contrary to a previous study done in a group of sickle cell disease patients in whom lower mean morphine consumption in the PCA group resulted in adequate pain relief and significantly less nausea and vomiting compared with the continuous infusion group during vaso-occlusive crises [13]. Recently this trend has changed, as consumption has been found to be equal or more compared to continuous infusion depending upon individual variability [6]. The fact that the amount of pethidine consumed was same in the two groups indicates the advantage offered to IV-PCA group patients, to take the dose of analgesia at the initial feeling of pain, leading to an improved analgesic profile with the same amount of opioid utilized.

Postoperative adverse events were minor in our study and consisted mainly of nausea and vomiting which was found more in continuous infusion group between 6 and $12 \mathrm{hrs}$. PONV is often associated with postoperative pain and its treatment [14] and we observed that it occurred at the time when patient had breakthrough pain and was given rescue boluses of pethidine.

We had prescribed metoclopramide routinely for every patient as per departmental protocol. Studies have proved that metoclopramide is ineffective in reducing the incidence and the severity of PONV [15]. The nausea and vomiting in our patients seems likely to be due to postoperative pain and its treatment and also due to the fact that multimodal anti-emetic was only administered on demand and not prophylactically on risk demand assessment [16].
The level of satisfaction was found to be higher in the IV-PCA group. It has been shown previously that effects of intravenous patient controlled analgesia and conventional intermittent or continuous opioid analgesia on intestinal function; recovery and length of hospital stay are nearly the same. However, patients using IV-PCA are more satisfied with their surgery and hospitalization than those who receive the conventional pain relief methods [17]. The benefit of PCA for the patient stems from prompt delivery of the analgesic, reducing the time between pain occurrence and drug administration $[18,19]$.

Although morphine remains the most important analgesic used in the management of postoperative pain [20], we used pethidine for both PCA and continuous infusion in our study. Developing countries face an acute shortage of opioids. Morphine is available in a very limited amount and pethidine is mostly used for postoperative pain management. In the literature, superior pain relief with morphine compared to pethidine has been demonstrated in two studies $[21,22]$, while other studies have failed to show any superiority of morphine $[23,24]$. In one study [25] PCA morphine and pethidine were equally effective for controlling postoperative pain and had similar side effects. Like any other opioid, pethidine is associated with certain unwanted effects, both for the mother and the newborn. The American Academy of Pediatrics Committee on Drugs labels pethidine in category 3 (compatible with breast feeding) [26]. This statement however comes with recommendation that infants should be watched for signs and symptoms of pethidine accumulation. In our study, we did not plan to observe the neonatal effects of pethidine in these breast feeding mothers, which is one of the limitations of our study.

Another limitation of our study is the use of pethidine as our primary analgesic agent, as pethidine is no longer used in many parts of the world for postoperative analgesia. Still there are many developing countries which are using pethidine like, one study done in rural health district of South Africa [27] has shown pethidine as the most common opioid prescribed (69\% of the patients) after CS and intramuscular route was the only route of administration. Our study in spite of this limitation can be helpful to many readers from the developing countries that are still faced with the limited availability of the opioids.

One of the other limitations of our study was our inability to do blinding. Since NRS for pain, need for rescue analgesia, total opioid consumption over 24 hours and observation of side effects in both groups were required to be assessed and documented, it was not possible to blind the assessor to the treatment group. We tried to remove the element of bias by assigning the pain nurses from AMPS who are trained and as part of their 
job description routinely do assessment for every postoperative patient under the APMS service. The AMPS nurses who were assessing, documenting and following the orders according to the protocol were not part of the research project. Two of the investigators after checking the pro forma for its completeness handed it over to the research officer for data entry.

We have used co-analgesics in our study, as patients in both group received diclofenac rectally every 8 hours and paracetamol orally every 6 hours. Siddik SM and colleagues [28] in their study have found improved analgesia and morphine sparing effect with the use of rectal diclofenac but they used intravenous paracetamol instead of oral.

Since we have obtained clearly positive results in favour of IV-PCA, we plan to share our results with the hospital administration and highlight the importance of obtaining IV-PCA pumps and thus slowly build up our resources in an endeavor to improve postoperative pain management after cesarean section in our patient population.

Since we have erratic and limited supply of opioids in our country, we can utilize other recourses including intravenous paracetamol [7] multimodal techniques like local analgesia, non-steroidal anti-inflammatory cocktail wound infiltration and abdominal nerve blocks as adjuncts [29] to postoperative analgesic regimes. This would benefit our caesarean section patients in reducing opioid consumption while providing better pain relief.

In conclusion postoperative pain management was found to be better with IV-PCA as compared to continuous opioid infusion. We need to promote IV-PCA in developing countries for the better pain control and patient satisfaction. We also believe that issue of cost and availability of drugs remains a challenge for effective pain management in developing countries. However we need to explore the possibility of enhancement of postoperative pain relief methods through alteration in the standard methods.

\section{REFERENCES}

[1] M. P. Bonnet, A. Mignon, J. X. Mazoit, Y. Ozier and E. Marret, "Analgesic Effect and Adverse Effects of Epidural Morphine Compared to Parenteral Opioids after Elective Caesarean Section: A Systemic Review,” European Journal of Pain, Vol. 14, No. 9, 2010, pp. 894-899. doi:10.1016/j.ejpain.2010.03.003

[2] D. A. Graves, R. L. Batenhorst, R. L. Bennett, et al., "Morphine Requirements Using Patient-Controlled Analgesia: Influence of Diurnal Variation and Morbid Obesity," Clinical Pharmacology, Vol. 2, No. 1, 1983, pp. 49-53.

[3] American Society of Anesthesiologists Task Force on Pain Management, Acute Pain Section, "Practice Guide- lines for Acute Pain Management in the Perioperative Setting," Anesthesiology, Vol. 82, No. 4, 1995, pp. 10711081. doi:10.1097/00000542-199504000-00032

[4] M. Silvasti, N. Svartling, M. Pitkanen and P. H. Rosenberg, "Comparison of Intravenous Patient Controlled Analgesia with Tramadol versus Morphine after Microvascular Breast Reconstruction,” European Journal of Anaesthesiology, Vol. 17, No. 7, 2000, pp. 448-555.

[5] M. J. Paech, J. S. Moore and S. F. Evans, "Meperidine for Patient-Controlled Analgesia after Cesarean Section. Intravenous versus Epidural Administration," Anesthesiology, Vol. 80, No. 6, 1994, pp. 1268-1276. doi:10.1097/00000542-199406000-00014

[6] K. A. Lehmann, "Recent Developments in Patient-Controlled Analgesia," Journal of Pain and Symptom Management, Vol. 29, No. 5, 2005, pp. 72-89. doi:10.1016/j.jpainsymman.2005.01.005

[7] H. Owen, J. L. Plummer, I. Armstrong, L. E. Mather and M. J. Cousins, "Variables of Patient-Controlled Analgesia: 1. Bolus Size,” Anaesthesia, Vol. 44, No. 1, 1989, pp. 710. doi:10.1111/j.1365-2044.1989.tb11087.x

[8] J. Tsang and B. Brush, "Patient-Controlled Analgesia in Postoperative Cardiac Surgery,” Anaesthesia and Intensive Care, Vol. 27, 1999, pp. 464-470.

[9] J. J. Church, "Continuous Narcotic Infusions for Relief of Postoperative Pain,” British Medical Journal, Vol. 1, 1979, pp. 977-979. doi:10.1136/bmj.1.6169.977

[10] J. V. Stapleton, K. L. Austin and L. E. Mather, “A Pharmacokinetic Approach to Postoperative Pain: Continuous Infusion of Pethidine," Anaesthesia and Intensive Care, Vol. 7, No. 1, 1979, pp. 25-32.

[11] Y. Al-Tamimi, K. F. Ilett, M. J. Paech, S. J. O’Halloran and P. E. Hartmann, "Estimation of Infant Dose and Exposure to Pethidine and Norpethidine via Breast Milk Following Patient-Controlled Epidural Pethidine for Analgesia Post Caesarean Delivery,” International Journal of Obstetric Anesthesia, Vol. 20, No. 2, 2011, pp. 128134. doi:10.1016/j.ijoa.2010.12.004

[12] J. A. Alhashemi, Q. A. Alotaibi, M. S. Mashaat, M. T. Kaid, R. H. Mujallid and M. A. Kaki, "Intravenous Acetaminophen vs Oral Ibuprofen in Combination with Morphine PCIA after Cesarean Delivery," Canadian Journal of Anesthesia, Vol. 53, No. 12, 2006, pp. 12001206. doi:10.1007/BF03021581

[13] E. J. Van Beers, C. F. Van Tuijn, P. T. Nieuwkerk, P. W. Friederich, J. H. Vranken and B. J. Biemond, "Pa- tientControlled Analgesia versus Continuous Infusion of Morphine during Vaso-Occlusive Crisis in Sickle Cell Disease, a Randomized Controlled Trial,” American Journal of Hematology, Vol. 82, No. 11, 2007, pp. 955-960. doi:10.1002/ajh.20944

[14] C. Simanski, H. H. Waldvogel and E. Neugebauer, "Postoperative Nausea and Vomiting (PONV). Clinical Significance, Basic Principles, Prevention and Therapy," Chirurg, Vol. 72, No. 12, 2001, pp. 1417-1426.

[15] L. H. Eberhart, W. Seeling, B. Ulrich, A. M. Morin and M. Georgieff, "Dimenhydrinate and Metoclopramide Alone or in Combination for Prophylaxis of PONV," Canadian 
Journal of Anesthesia, Vol. 47, 2000, pp. 780-785. doi:10.1007/BF03019481

[16] D. Rüsch, K. Becker, L. H. Eberhart, et al., "Postoperative Nausea and Vomiting (PONV)-Recommendations for Risk Assessment, Prophylaxis and Therapy-Results of an Expert Panel Meeting," Anasthesiologie, Intensivmedizin, Notfallmedizin, Schmerztherapie, Vol. 46, No. 3, 2011, pp. 158-170.

[17] C. H. Shu, Y. F. Liu, C. C. Tsou, Y. J. Hu and Y. C. Kuo, "Patient Controlled Analgesia Application to the Large Intestine Rectum Surgery,” Taiwan Medical Journal, Vol. 43, 2000, pp. 15-16.

[18] P. E. Macintyre, "Safety and Efficacy of Patient Controlled Analgesia," British Journal of Anaesthesia, Vol. 87, 2001, pp. 36-46. doi:10.1093/bja/87.1.36

[19] C. Mann, F. Ouro-Bang'na and J. J. Eledjam, “PatientControlled Analgesia,” Current Drug Targets, Vol. 6, 2005, pp. 815-819.

[20] G. Smith, “Pain after Surgery (Editorial),” British Journal of Anaesthesia, Vol. 67, 1991, pp. 233-234. doi:10.1093/bja/67.3.233

[21] R. S. Sinatra, K. Lodge, K. Sibert, K. S. Chung, J. H. Chung, A. Parker and D. M. Harison, “A Comparison of Morphine, Meperidine, and Oxymorphone as Utilized in Patient Controlled Analgesia Following Cesarean Delivery,” Anesthesiology, Vol. 70, No. 4, 1989, pp. 585-590. doi:10.1097/00000542-198904000-00005

[22] M. Bahar, M. Rosen and M. D. Vickers, "Self-Administered Nalbuphine, Morphine and Pethidine. Comparison, by Intravenous Route, Following Cholecystectomy,” Anaesthesia, Vol. 40, No. 6, 1985, pp. 529-532.

[23] A. Woodhouse, A. F. T. Hobbes, L. E. Mather and M.
Gibson, “A Comparison of Morphine, Pethidine, and Fentanyl in the Post-Surgical Patient-Controlled Analgesia Environment,” Pain, Vol. 64, No. 1, 1996, pp. 115-121. doi:10.1016/0304-3959(95)00082-8

[24] R. McKenzie, T. Rudy and M. Ponter-Hammill, "SideEffects of Morphine Patient-Controlled Analgesia and Meperidine Patient Controlled Analgesia: A Follow up of 500 Patients," Journal of the American Association of Nurse Anesthetists, Vol. 60, No. 3, 1992, pp. 282-286.

[25] H. Unlugenc, M. A. Vardar and S. Tetiker, “A Comparative Study of the Analgesic Effect of Patient-Controlled Morphine, Pethidine, and Tramadol for Postoperative Pain Management after Abdominal Hysterectomy," Anesthesia \& Analgesia, Vol. 106, No. 1, 2008, pp. 309-312.

[26] American Academy of Pediatrics, Committee on Drugs, "Transfer of Drugs and Other Chemicals into Human Milk,” Pediatrics, Vol. 108, No. 3, 2001, pp. 776-789.

[27] M. F. Khan, O. B. Omole and G. J. O. Marincowitz, "Postoperative Analgesia Following Caesarean Deliveries in a Rural Health District of South Africa," Tropical Doctor, Vol. 39, No. 4, 2009, pp. 217-221. doi:10.1258/td.2009.070497

[28] S. M. Siddik, M. T. Aouad, M. I. Jalbout, L. B. Rizk, G. H. Kamar and A. S. Baraka, "Diclofenac and/or Propacetamol for Postoperative Pain Management after Cesarean Delivery in Patients Receiving Patient Controlled Analgesia Morphine," Regional Anesthesia and Pain Medicine, Vol. 26, 2001, pp. 310-315.

[29] B. A. Amigboye and G. J. Hofmeyr, "Local Anaesthetic Wound Infilteration and Abdominal Nerves Block during Caesarean Section for Postoperative Pain Relief," Cochrane Database of Systematic Reviews, Vol. 3, 2009, Article ID: CD006954. 